\title{
ARTICLE
}

Chronic lymphocytic leukemia

\section{Obinutuzumab plus bendamustine in previously untreated patients with CLL: a subgroup analysis of the GREEN study}

\author{
Stephan Stilgenbauer ${ }^{1,2} \cdot$ Veronique Leblond $^{3} \cdot$ Robin Foà $^{4} \cdot$ Sebastian Böttcher ${ }^{5,6} \cdot$ Osman Ilhan $^{7} \cdot$ \\ Wolfgang Knauf $^{8}$ - Eva Mikuskova ${ }^{9} \cdot$ Christoph Renner $^{10}$ - Eugen Tausch ${ }^{1}$ - Dariusz Woszczyk ${ }^{11,12}$. \\ Ekaterina Gresko ${ }^{13} \cdot$ Linda Lundberg $^{13} \cdot$ Tom Moore $^{13} \cdot$ Thea Morris $^{13} \cdot$ Susan Robson ${ }^{13} \cdot$ Francesc Bosch $^{14}$
}

Received: 22 November 2017 / Revised: 13 March 2018 / Accepted: 28 March 2018 / Published online: 27 April 2018

(c) The Author(s) 2018. This article is published with open access

\begin{abstract}
GREEN (NCT01905943) is a non-randomized, open-label phase IIIb study investigating obinutuzumab alone or plus chemotherapy in chronic lymphocytic leukemia (CLL). We report a preplanned subgroup analysis of 158 previously untreated CLL patients receiving obinutuzumab-bendamustine (G-B). Patients received six 28-day cycles (C) of G-B: obinutuzumab day (D)1/D2 of C1 (25 mg D1/975 mg D2), $1000 \mathrm{mg} \mathrm{D} 8$ and D15 of C1, and D1 of C2-6; and bendamustine 70/90 mg/m² D1 and D2 of C1-6. The primary endpoint was safety/tolerability. Grade $\geq 3$ adverse events (AEs) occurred in $82.3 \%$ of patients, including neutropenia (49.4\%), thrombocytopenia (12.0\%) and febrile neutropenia (10.8\%). Serious AEs included neutropenia $(12.7 \%)$, febrile neutropenia $(9.5 \%)$ and pneumonia $(7.6 \%)$. Rates of grade $\geq 3$ infections and infusion-related reactions were $20.3 \%$ and $17.1 \%$, respectively. Due to tumor lysis syndrome (TLS; $8.2 \%$ ), including two associated fatalities (one in another study cohort), additional risk-minimization measures were implemented. Overall response rate was $81.0 \%$. After 32.8 months' median observation time, 2-year progression-free survival was $81.8 \%$. Minimal residual disease was undetectable in 59.5\% (94/ 158 ) and $27.8 \%$ (44/158) of patients for blood and bone marrow, respectively. Frontline G-B appears to have manageable toxicity with clinical activity in CLL. Careful TLS risk assessment, pretreatment and monitoring is required.
\end{abstract}

Electronic supplementary material The online version of this article (https://doi.org/10.1038/s41375-018-0146-5) contains supplementary material, which is available to authorized users.

Stephan Stilgenbauer

Stephan.Stilgenbauer@uniklinik-ulm.de

1 Department of Internal Medicine III, Ulm University, Ulm, Germany

2 Present address: Klinik für Innere Medizin I, Universitätsklinikum des Saarlandes, Homburg, Germany

3 UPMC GRC11-GRECHY, AP-HP Hôpital Pitié Salpêtrière, Paris, France

4 Department of Cellular Biotechnologies and Hematology, 'Sapienza' University, Rome, Italy

5 Second Department of Medicine, University of Schleswig-Holstein, Campus Kiel, Kiel, Germany

\section{Introduction}

Chronic lymphocytic leukemia (CLL) is the most common adult leukemia in the western world [1]. The standard firstline treatment for physically fit CLL patients is chemoimmunotherapy with fludarabine-cyclophosphamiderituximab (R-FC) [2-6]. However, many CLL patients are elderly with comorbidities and ineligible for fludarabine-

6 Clinic III, Hematology, Oncology and Palliative Medicine, University of Rostock, Rostock, Germany

7 Ankara University, Ankara, Turkey

8 Onkologische Gemeinschaftspraxis, Agaplesion Bethanien Krankenhaus, Frankfurt, Germany

9 National Cancer Institute, Bratislava, Slovakia

10 OnkoZentrum Zürich, Zürich, Switzerland

11 State Hospital, Opole, Poland

12 Present address: Haematology Department, University of Opole, Provincial Hospital, Opole, Poland

13 F. Hoffmann-La Roche Ltd, Basel, Switzerland

14 University Hospital Vall d'Hebron, Barcelona, Spain 
based treatment $[7,8]$. New combinations with less toxicity and alternative chemotherapy backbones are needed.

Chemoimmunotherapy with rituximab-bendamustine (R-B) is effective and well tolerated in fit patients with previously untreated or relapsed/refractory CLL [9, 10], although its efficacy was inferior to R-FC in the phase III CLL10 study [5]. The pivotal CLL11 study demonstrated that the glycoengineered, type II anti-CD20 antibody obinutuzumab (GA101) was superior to rituximab (combined with chlorambucil; G-Clb vs $\mathrm{R}-\mathrm{Clb}$ ) in treatment-naive patients with CLL and coexisting conditions [11]. Thus, there is a rationale for evaluating obinutuzumab-bendam ustine (G-B) in CLL.

GREEN (NCT01905943) is an ongoing phase IIIb study investigating obinutuzumab alone or with chemotherapy in previously untreated or relapsed/refractory CLL [12]. Here, we report on a preplanned safety and efficacy analysis from a subgroup of previously untreated CLL patients who received G-B in GREEN.

\section{Patients and methods}

\section{Study design}

GREEN is a non-randomized, open-label, non-comparative, multicenter study in previously untreated or relapsed/ refractory CLL. The primary objective is to evaluate the safety/tolerability of obinutuzumab alone or combined with various chemotherapy regimens; the secondary objective is to assess efficacy. The exploratory objective is to investigate alternative obinutuzumab administration measures to mitigate/reduce infusion-related reactions (IRRs). For this, patients included in GREEN were assigned to three different cohorts (Supplementary Figure S1).

Planned analyses for GREEN were to report safety and efficacy summaries for each treatment regimen separately as well as pooled overall safety. Here, we report the subgroup of previously untreated patients receiving G-B assigned to cohort 1 of GREEN (trial fully accrued since 30 March 2016 (first enrolled, 24 October 2013), with follow-up ongoing), who received a modified obinutuzumab dosing regimen to mitigate IRRs: the initial dose was given in two parts on consecutive days $(25 \mathrm{mg}$ on day (D) 1 of cycle (C) 1 at an infusion rate of $12.5 \mathrm{mg} / \mathrm{h}$, followed by $975 \mathrm{mg}$ on D2 at an initial rate of $50 \mathrm{mg} / \mathrm{h}$, then $1000 \mathrm{mg}$ on D8 and D15 of C1, and D1 of C2-6 as standard).

Chemotherapy options (28-day cycles) in all cohorts were partly dependent on patient fitness and based on investigator choice (Supplementary Figure S1). Bendamustine was dosed at $90 \mathrm{mg} / \mathrm{m}^{2}$ (or $70 \mathrm{mg} / \mathrm{m}^{2}$ in unfit patients at the investigator's discretion) on D1 and D2 of C1-6. Granulocyte colony-stimulating factor (G-CSF) use was permitted; primary prophylaxis was advised for patients aged $\geq 60$ years and/or with comorbidities.

Risk-minimization measures (including adequate hydration (fluid intake $31 /$ day, starting 1-2 days before first obinutuzumab dose) and allopurinol (or similar e.g. rasburicase) prophylaxis for at least $72 \mathrm{~h}$ prior to first obinutuzumab dose) for tumor lysis syndrome (TLS) [13], an identified risk in patients treated with obinutuzumab, were included in the protocol from the start. Patients were considered at risk of TLS if they had node(s) $\geq 10 \mathrm{~cm}$; or node(s) $\geq 5 \mathrm{~cm}$ and $<10$ $\mathrm{cm}$, and an absolute lymphocyte count (ALC) $\geq 25 \times 10^{9} / 1$ or renal impairment (creatinine clearance $(\mathrm{CrCl})<70 \mathrm{ml} / \mathrm{min}$ ); or ALC $\geq 25 \times 10^{9} / \mathrm{l}$ and $\mathrm{CrCl}<70 \mathrm{ml} / \mathrm{min}$. After two reports of fatal TLS cases in G-B-treated patients (including one in cohort 1), additional risk-minimization measures, including investigator training to emphasize the importance of TLS prophylaxis, were instigated for all cohorts, as described in the supplement. However, these additional measures did not apply to patients in cohort 1 as they had already completed treatment.

GREEN was conducted in accordance with the Declaration of Helsinki, Good Clinical Practice Guidelines and local laws and was approved by institutional review boards/ethics committees at participating centers. Patients provided written informed consent. All authors had access to the primary clinical data and were involved in the data analysis.

\section{Patient population}

Cohort 1 enrolled previously untreated CLL patients only. Inclusion criteria were: age $\geq 18$ years; requiring treatment per International Workshop on CLL (iwCLL) criteria; [14] adequate hematologic function; and Eastern Cooperative Oncology Group performance status $\leq 2$. Patients with a $17 \mathrm{p}$ deletion could be enrolled; those with severe renal impairment $(\mathrm{CrCl}<30 \mathrm{ml} / \mathrm{min})$ were excluded. Full inclusion/exclusion criteria for previously untreated CLL patients (cohorts 1-3) are detailed in the supplement.

\section{Study endpoints}

The primary endpoint of GREEN was safety/tolerability. Secondary endpoints were overall response rate (ORR) including complete response (CR), progressionfree survival (PFS) and minimal residual disease (MRD). 


\section{Assessments}

\section{Safety}

Adverse events (AEs), serious AEs (SAEs) and AEs of special or particular interest (AESI/AEPI) were monitored and graded using National Cancer Institute Common Terminology Criteria for AEs version 4.0. IRRs were defined as any $\mathrm{AE}$ occurring during or within $24 \mathrm{~h}$ of obinutuzumab infusion and considered related to obinutuzumab.

\section{Efficacy}

ORR was assessed by the investigator per iwCLL criteria [14] at the 'final response assessment' visit (scheduled 84 days after last treatment dose). A computed tomography (CT) scan was required to confirm $\mathrm{CR}$ and partial response (PR); patients lacking a valid CT scan were classed as stable disease (SD). A bone marrow biopsy was required for confirmation of CR; patients otherwise meeting CR criteria but lacking a valid biopsy were classed as PR. Other efficacy assessments including PFS, MRD measurement and analysis of prognostic markers are detailed in the supplement.

\section{Statistical methodology}

Details of the sample size estimation are provided in the supplement. Safety variables were analyzed in the safety population $(N=158)$, i.e., all patients who received at least one dose of study treatment. Efficacy variables were analyzed in the intent-to-treat (ITT) population $(N=158)$, comprising all patients from cohort 1 who received at least a partial dose of both obinutuzumab and bendamustine (data cut-off, 29 December 2016). For the MRD analyses, the 'intent-to-ship' population $(N=140)$ comprised all patients whose MRD samples at the final response assessment could be shipped to the central laboratory within $48 \mathrm{~h}$ (thus excluding Argentina, Brazil, Canada, China, Korea, Mexico, Thailand and Uruguay). The 'MRD-evaluable' population $(N=105)$ comprised all patients with an available MRD result (blood or bone marrow).

For ORR, patients with a missing response assessment during the defined time window (D56-168 after last treatment dose) were considered non-responders. Response rates were presented with two-sided, 95\% Clopper-Pearson confidence intervals (CIs). Estimates for the survival functions for PFS were obtained using the Kaplan-Meier approach. After the data snapshot was taken for analysis, a further 5 AEs related to 4 patients in this subgroup were reported late by the sites on the database that remained open to continue collecting information until the final analysis. In addition, one patient with SD at the
Table 1 Baseline patient characteristics in patients receiving G-B in cohort 1 of the GREEN study (intent-to-treat population)

\begin{tabular}{|c|c|}
\hline Characteristic & $\begin{array}{l}\text { All patients } \\
(N=158)\end{array}$ \\
\hline $\begin{array}{l}\text { Median age, years } \\
\text { (range) }\end{array}$ & $69.0(42-83)$ \\
\hline Male/female, $n(\%)$ & $103 / 55(65.2 / 34.8)$ \\
\hline CIRS $>6, n(\%)$ & $28(17.7)$ \\
\hline $\mathrm{CrCl}<70 \mathrm{ml} / \mathrm{min}, n(\%)$ & $73(46.2)$ \\
\hline $\mathrm{CrCl}<50 \mathrm{ml} / \mathrm{min}, n(\%)$ & $21(13.3)$ \\
\hline $\begin{array}{l}\mathrm{CIRS}>6 \text { and } \mathrm{CrCl}<70 \\
\mathrm{ml} / \mathrm{min}, n(\%)\end{array}$ & $13(8.2)$ \\
\hline \multicolumn{2}{|l|}{ Fitness, $n(\%)$} \\
\hline $\mathrm{Fit}^{\mathrm{a}}$ & $70(44.3)$ \\
\hline Unfit $^{\mathrm{b}}$ & $88(55.7)$ \\
\hline \multicolumn{2}{|c|}{ Binet stage at screening, $n(\%)$} \\
\hline A & $48(30.4)$ \\
\hline B & $57(36.1)$ \\
\hline $\mathrm{C}$ & $53(33.5)$ \\
\hline \multicolumn{2}{|c|}{ Absolute lymphocyte count, $N=155, n(\%)$} \\
\hline$\geq 50 \times 10^{9} / 1$ & $88(56.8)$ \\
\hline \multicolumn{2}{|c|}{ Tumor bulk, $N=139, n(\%)$} \\
\hline$\geq 5 \mathrm{~cm}$ & $95(68.3)$ \\
\hline \multicolumn{2}{|c|}{ Genomic aberrations, $N=146, n(\%)^{\mathrm{c}}$} \\
\hline $17 \mathrm{p}$ deletion & $11(7.5)$ \\
\hline 11q deletion & $26(17.8)$ \\
\hline $12 q$ trisomy & $26(17.8)$ \\
\hline $13 q$ deletion & $52(35.6)$ \\
\hline Other abnormality & $6(4.1)$ \\
\hline Normal & $25(17.1)$ \\
\hline \multicolumn{2}{|l|}{$\mathrm{IGHV}, N=136, n(\%)$} \\
\hline Unmutated & $92(67.6)$ \\
\hline Mutated & $44(32.4)$ \\
\hline \multicolumn{2}{|l|}{$\mathrm{ZAP} 70, N=129, n(\%)$} \\
\hline Positive & $82(63.6)$ \\
\hline Negative & $47(36.4)$ \\
\hline \multicolumn{2}{|l|}{$\mathrm{CD} 38, N=129, n(\%)$} \\
\hline Positive & $70(54.3)$ \\
\hline Negative & $59(45.7)$ \\
\hline
\end{tabular}

CIRS Cumulative Illness Rating Scale, $\mathrm{CrCl}$ creatinine clearance, $G-B$ obinutuzumab plus bendamustine, IGHV immunoglobulin heavy variable chain

${ }^{\text {a }} \mathrm{CIRS} \leq 6$ and $\mathrm{CrCl} \geq 70 \mathrm{ml} / \mathrm{min}$

${ }^{\mathrm{b}}$ CIRS $>6$ and/or $\mathrm{CrCl}<70 \mathrm{ml} / \mathrm{min}$

${ }^{c}$ According to the hierarchical model of genomic aberrations [29]

final response assessment had this changed to PR. These updates are not part of the statistical analysis summary tables and listings presented. Please see Supplementary Tables S1 and S2. 


\section{Results}

\section{Patient, treatment exposure and observation time}

The ITT population comprised 158 patients with CLL (70 fit, 88 unfit) (Table 1; Supplementary Figure S2). Mean number of obinutuzumab administrations was 8.4 (planned, 9) and $149(94.3 \%)$ patients received $\geq 90 \%$ of the planned dose (based on actual/planned doses). Mean number of bendamustine cycles was 5.3 (planned, 6), with 142 $(89.9 \%)$ patients receiving $\geq 90 \%$ of the planned dose $(68$ patients received bendamustine $70 \mathrm{mg} / \mathrm{m}^{2}$ and 90 patients received $90 \mathrm{mg} / \mathrm{m}^{2}$ ). Median observation time was 32.8 (range, 0.5-37.5) months.

\section{Safety}

Most patients $(96.8 \%, 153 / 158)$ reported at least one AE; neutropenia (62.7\%), pyrexia $(41.8 \%)$ and thrombocytopenia $(32.3 \%)$ were the most frequent. Overall, $88.6 \%$ of patients experienced at least one treatment-related $\mathrm{AE}$, most commonly neutropenia (57.0\%), pyrexia $(31.0 \%)$ and thrombocytopenia (29.1\%). Twenty-six patients $(16.5 \%)$ discontinued at least one study treatment prematurely due to an AE, 11 (7.0\%) of whom did so because of neutropenia. G-CSF was administered to $107 / 158$ $(67.7 \%)$ patients at least once during the study. Common grade $\geq 3$ AEs, serious AEs and grade $\geq 3$ AESI are summarized in Table 2.

IRRs occurred in $57.6 \%(n=91)$ of patients; $17.1 \%$ $(n=27)$ experienced grade $\geq 3$ IRRs. Serious IRRs occurred in $10.1 \%(n=16)$ of patients (no fatal cases). Infections occurred in $54.4 \%(n=86)$ of patients; $20.3 \%$ reported grade $\geq 3$ infection. Grade $\geq 3$ infections (by preferred term) in more than one patient were: pneumonia $(n=12)$; sepsis and urinary tract infection ( $n=3$ each); and cytomegaloviral pneumonia, erysipelas, herpes zoster and lung infection $(n=2$ each). Serious infections occurred in $19.6 \%$ of patients, including ( $\geq 1 \%)$ : pneumonia $(n=12)$; sepsis $(n=3)$; and herpes zoster, lung infection, cytomegaloviral pneumonia and urinary tract infection $(n=2$ each). Grade $\geq 3$ AEs related to neutropenia were reported by $53.2 \%$ of patients. TLS (grade $\geq 3$ ) was observed in $13(8.2 \%$ ) patients, and included one fatality. TLS was one of the most common SAEs $(n=6 ; 3.8 \%)$ by preferred term, along with neutropenia $(12.7 \%)$, febrile neutropenia $(9.5 \%)$, pneumonia $(7.6 \%)$ and pyrexia $(7.0 \%)$ (Table 2$)$.

The tolerability of G-B appeared more favorable in fit vs unfit patients based on rates of grade $\geq 3$ AEs and SAEs (Table 2). Rates of grade $\geq 3$ TLS, infections and IRRs were numerically lower in fit vs unfit patients. Serious TLS events occurred in $6.8 \%$ of unfit patients, but were not observed in fit patients.
Table 2 Grade 3 or higher adverse events and serious adverse events in patients receiving G-B in cohort 1 of the GREEN study (safety population)

\begin{tabular}{|c|c|c|c|}
\hline$n(\%)$ & $\begin{array}{l}\text { All patients } \\
(N=158)\end{array}$ & $\begin{array}{l}\text { Fit patients }{ }^{\mathrm{a}} \\
(n=70)\end{array}$ & $\begin{array}{l}\text { Unfit patients }^{\mathrm{b}} \\
(n=88)\end{array}$ \\
\hline \multicolumn{4}{|c|}{$\begin{array}{l}\text { Grade } \geq 3 \text { AEs (reported by } \geq 2 \% \text { patients in overall population) by preferred } \\
\text { term }\end{array}$} \\
\hline Any & $130(82.3)$ & $52(74.3)$ & 78 (88.6) \\
\hline Neutropenia & $78(49.4)$ & 34 (48.6) & $44(50.0)$ \\
\hline Thrombocytopenia & $19(12.0)$ & $8(11.4)$ & $11(12.5)$ \\
\hline Febrile neutropenia & $17(10.8)$ & $8(11.4)$ & $9(10.2)$ \\
\hline Lymphopenia & $14(8.9)$ & $6(8.6)$ & $8(9.1)$ \\
\hline Anemia & $13(8.2)$ & $3(4.3)$ & $10(11.4)$ \\
\hline Leukopenia & $13(8.2)$ & $6(8.6)$ & $7(8.0)$ \\
\hline Tumor lysis syndrome & $13(8.2)$ & $2(2.9)$ & $11(12.5)$ \\
\hline Pneumonia & $12(7.6)$ & $5(7.1)$ & $7(8.0)$ \\
\hline Hypertension & $11(7.0)$ & $5(7.1)$ & $6(6.8)$ \\
\hline Hyperglycemia & $6(3.8)$ & $4(5.7)$ & $2(2.3)$ \\
\hline Squamous cell carcinoma & $5(3.2)$ & $1(1.4)$ & $4(4.5)$ \\
\hline Diarrhea & $4(2.5)$ & 0 & $4(4.5)$ \\
\hline Hyperuricemia & $4(2.5)$ & $4(5.7)$ & 0 \\
\hline \multicolumn{4}{|c|}{ Serious AEs (reported by $\geq 2 \%$ patients in overall population) by preferred tern } \\
\hline Any & $96(60.8)$ & $37(52.9)$ & $59(67.0)$ \\
\hline Neutropenia & 20 (12.7) & $9(12.9)$ & $11(12.5)$ \\
\hline Febrile neutropenia & $15(9.5)$ & $6(8.6)$ & $9(10.2)$ \\
\hline Pneumonia & $12(7.6)$ & $5(7.1)$ & $7(8.0)$ \\
\hline Pyrexia & $11(7.0)$ & $4(5.7)$ & $7(8.0)$ \\
\hline Tumor lysis syndrome & $6(3.8)$ & 0 & $6(6.8)$ \\
\hline Squamous cell carcinoma & $4(2.5)$ & $1(1.4)$ & $3(3.4)$ \\
\hline Thrombocytopenia & $4(2.5)$ & $1(1.4)$ & $3(3.4)$ \\
\hline \multicolumn{4}{|l|}{ Grade $\geq 3 \mathrm{AESI} / \mathrm{AEPI}^{\mathrm{c}}$} \\
\hline Neutropenia & $84(53.2)$ & $37(52.9)$ & $47(53.4)$ \\
\hline Infections & $32(20.3)$ & $11(15.7)$ & $21(23.9)$ \\
\hline IRRs & $27(17.1)$ & $9(12.9)$ & $18(20.5)$ \\
\hline Thrombocytopenia & $19(12.0)$ & $8(11.4)$ & $11(12.5)$ \\
\hline Second malignancies & $13(8.2)$ & $4(5.7)$ & $9(10.2)$ \\
\hline Tumor lysis syndrome & $13(8.2)$ & $2(2.9)$ & $11(12.5)$ \\
\hline
\end{tabular}

$A E$ adverse event, AEPI adverse events of particular interest, $A E S I$ adverse events of special interest, CIRS Cumulative Illness Rating Scale, $\mathrm{CrCl}$ creatinine clearance, $G-B$ obinutuzumab plus bendamustine, IRR infusion-related reaction, MedDRA Medical Dictionary for Regulatory Activities

${ }^{a} \mathrm{CIRS} \leq 6$ and $\mathrm{CrCl} \geq 70 \mathrm{ml} / \mathrm{min}$

${ }^{\mathrm{b}} \mathrm{CIRS}>6$ and/or $\mathrm{CrCl}<70 \mathrm{ml} / \mathrm{min}$

${ }^{c}$ IRRs were defined as any AE occurring during or within $24 \mathrm{~h}$ of obinutuzumab infusion and considered related to obinutuzumab; infection selection was via the MedDRA system order class 'Infections and Infestations'; second malignancy selection was via the MedDRA system organ class 'Neoplasms Benign, Malignant, and Unspecified' starting 6 months after the first study drug intake; neutropenia and thrombocytopenia selection was via their MedDRA basket dataset subgroups; and tumor lysis syndrome was defined by its preferred term

Seventeen deaths (10.8\%) were reported (fit $n=6$; unfit $n=11$ ), two of which occurred on, or within 28 days of last, study treatment; five deaths (all resulting from AEs) were considered related to treatment (West Nile virus infection, acute liver failure, febrile neutropenia with TLS, 
arrhythmia and brain metastasis of adenocarcinoma). Four deaths were due to disease progression and 13 were due to AEs (two with prior progressive disease (PD]) (Supplementary Table S3).

\section{Response assessment}

ORR at the final response assessment was $81.0 \%$ (95\% CI, 74.0-86.8; CR (including CR with incomplete marrow recovery (CRi)), $34.8 \%$; Table 3). Overall, $9.5 \%$ of patients had a missing assessment and were classed as nonresponders. ORR was broadly similar in unfit and fit patients (Table 3), and of those patients who reported TLS in the ITT population, $69.3 \%$ (9/13) responded to treatment. Response rates were also similar between bendamustine dose groups (70 mg/m²: ORR, 83.8\% (57/68; 95\% CI, 72.9-91.6); CR/CRi, $38.2 \%(26 / 68) ; 90 \mathrm{mg} / \mathrm{m}^{2}$ : ORR, $78.9 \%$ (71/90; 95\% CI, 69.0-86.8); CR/CRi, 32.2\% (29/90)).

Median PFS was not reached; at data cut-off, $23.4 \%$ of patients had experienced an event (Fig. 1a). Estimated PFS at 12 and 24 months was $92.3 \%$ and $81.8 \%$, respectively. PFS appeared similar in fit vs unfit patients (Fig. 1b). Despite the limited size of subgroups, there was a trend for shorter PFS in older patients ( $\geq 65$ years), and in patients with a $17 \mathrm{p}$ or $11 \mathrm{q}$ deletion, $12 \mathrm{q}$ trisomy, unmutated immunoglobulin heavy variable chain (IGHV) or CD38 ${ }^{+}$ CLL (Supplementary Table S4; Fig. 1c, d).

\section{MRD analysis at final response assessment}

For the ITT population, undetectable MRD $\left(<10^{-4}\right.$, i.e., MRD-negativity $\left(\mathrm{MRD}^{-}\right)$) was observed in 59.5\% (94/158) and $27.8 \%$ (44/158) for blood and bone marrow samples, respectively. $\mathrm{MRD}^{-}$rates in the intent-to-ship population were $67.1 \%$ (94/140; blood) and $31.4 \%$ (44/140; bone marrow). The MRD-evaluable population comprised 104

Table 3 Response in patients receiving G-B in cohort 1 of the GREEN study at final response assessment (intent-to-treat population)

\begin{tabular}{llll}
\hline Response, $n(\%)$ & $\begin{array}{l}\text { All patients } \\
(N=158)\end{array}$ & $\begin{array}{l}\text { Fit patients }^{\mathrm{a}} \\
(n=70)\end{array}$ & $\begin{array}{l}\text { Unfit patients }^{\mathrm{b}} \\
(n=88)\end{array}$ \\
\hline Overall response $^{\mathrm{C}}$ & $128(81.0)$ & $60(85.7)$ & $68(77.3)$ \\
Complete response $^{\mathrm{c}}$ & $55(34.8)$ & $23(32.9)$ & $32(36.4)$ \\
Partial response & $73(46.2)$ & $37(52.9)$ & $36(40.9)$ \\
Stable disease & $13(8.2)$ & $4(5.7)$ & $9(10.2)$ \\
$\begin{array}{l}\text { Progressive disease } \\
\text { Failure (due to } \\
\text { missing assessment) }\end{array}$ & $2(1.3)$ & $1(1.4)$ & $1(1.1)$ \\
\hline
\end{tabular}

CIRS Cumulative Illness Rating Scale, $\mathrm{CrCl}$ creatinine clearance, $G-B$ obinutuzumab plus bendamustine

${ }^{a} \mathrm{CIRS} \leq 6$ and $\mathrm{CrCl} \geq 70 \mathrm{ml} / \mathrm{min}$

${ }^{\mathrm{b}} \mathrm{CIRS}>6$ and/or $\mathrm{CrCl}<70 \mathrm{ml} / \mathrm{min}$

${ }^{\mathrm{c}}$ Including complete response with incomplete marrow recovery patients with an evaluable blood sample and 65 with an evaluable bone marrow sample (64 with blood and bone marrow and 1 with bone marrow only). $\mathrm{MRD}^{-}$rates in the MRD-evaluable population were $90.4 \%$ (94/104) and $67.7 \%(44 / 65)$ for blood and bone marrow, respectively (Table 4).

$\mathrm{MRD}^{-}$rates were similar for bendamustine dose groups: $92.3 \%$ (36/39; 95\% CI, 79.1-98.4) and 89.2\% (58/65; $95 \%$ CI, 79.1-95.6) for 70 and $90 \mathrm{mg} / \mathrm{m}^{2}$, respectively.

Patients who were MRD negative in blood at final response assessment had longer PFS than those who were not (Fig. 1e); however, this may be biased as all early withdrawals had no MRD assessment at the final response assessment by definition.

\section{Discussion}

In the phase Ib GALTON and phase II GIBB studies, G-B appeared to have acceptable toxicity and was active in previously untreated CLL patients $[15,16]$. Our subgroup analysis of a cohort of first-line patients receiving G-B indicates a similar trend in a larger international, multicenter study, and suggests that G-B may have manageable toxicity and promising efficacy in most patients. However, it also highlights the need for effective monitoring and management of TLS in G-B-treated patients. Thirteen (8.2\%) patients herein experienced TLS with one associated death, whereas the incidence of TLS with G-Clb in the pivotal CLL11 trial was $4 \%$ with no associated deaths [11]. With the development of increasingly efficacious treatments, recognition and management of TLS in CLL is of evergreater importance, especially when such treatments are given to patients with comorbidities, such as renal impairment. Specific guidance for the prevention and treatment of TLS and IRRs is provided in the labeling for obinutuzumab $[17,18]$, and a manuscript reporting the outcome of different IRR risk-mitigation strategies employed in GREEN will be published separately.

In addition, as detailed in the supplement, at-risk patients must be recognized prior to treatment initiation, receive appropriate prophylaxis and be monitored closely for occurrence of TLS signs during initial treatment. Any additional guidelines according to standard practice should be followed.

AEs were consistent with the known safety profile of obinutuzumab. Rapid B-cell depletion with obinutuzumab, accompanied by rapid release of cytokines including interleukin-6, interleukin-8 and tumor necrosis factor alpha, may explain the frequency and intensity of IRRs with obinutuzumab vs rituximab $[11,19]$. Grade $\geq 3$ IRRs developed in $20.5 \%$ of unfit patients (17.1\% of G-B patients overall), which was comparable with that $(20 \%)$ reported for $\mathrm{G}-\mathrm{Clb}$ 
a

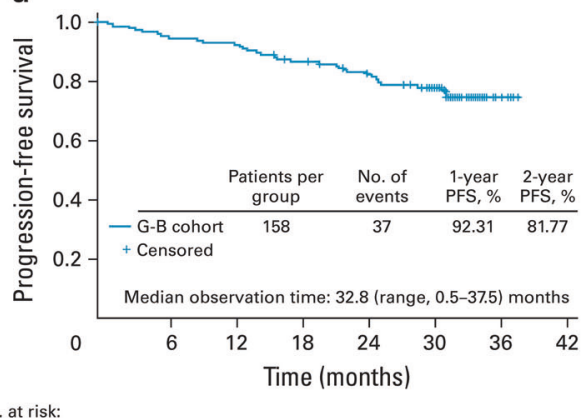

b

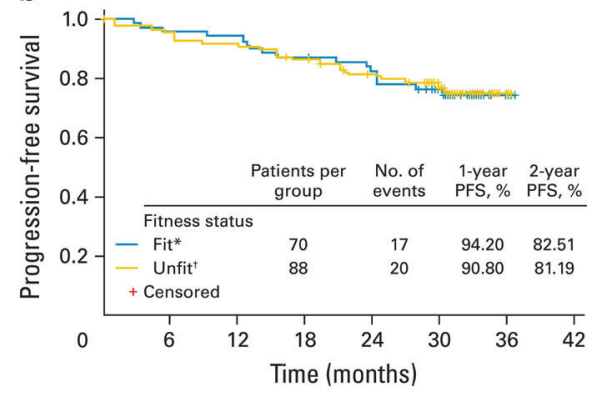

No. at risk:

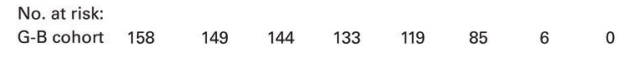

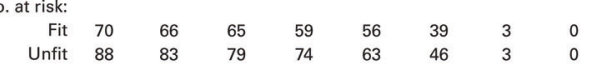

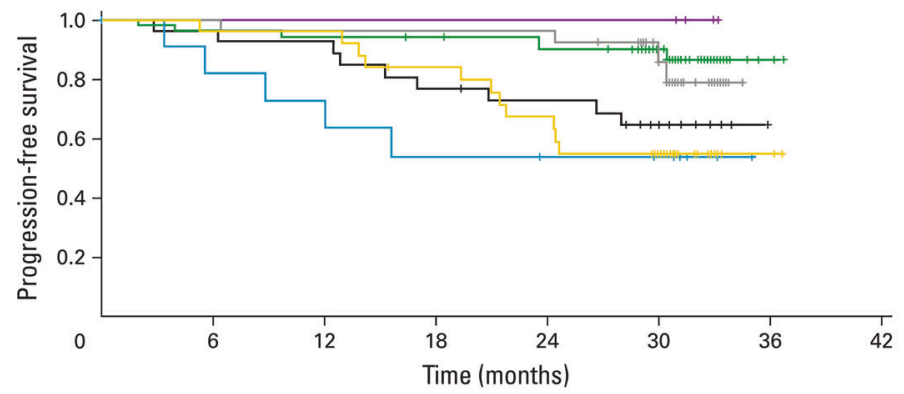

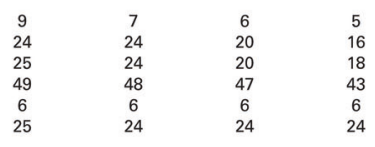

$\begin{array}{cc}5 & \\ 16 & 10 \\ 18 & 12 \\ 43 & 33 \\ 6 & \\ 24 & 14\end{array}$

$\begin{array}{ccc}4 & 0 & 0 \\ 10 & 2 & 0 \\ 12 & 0 & 0 \\ 33 & 4 & 0 \\ 6 & 0 & 0 \\ 14 & 0 & 0\end{array}$

d

$\begin{array}{rllllllll}\text { No. at risk: } & & & & & & & & \\ \text { Mutated } & 44 & 40 & 39 & 38 & 34 & 25 & 1 & 0 \\ \text { Unmutated } & 92 & 88 & 85 & 77 & 70 & 48 & 4 & 0\end{array}$

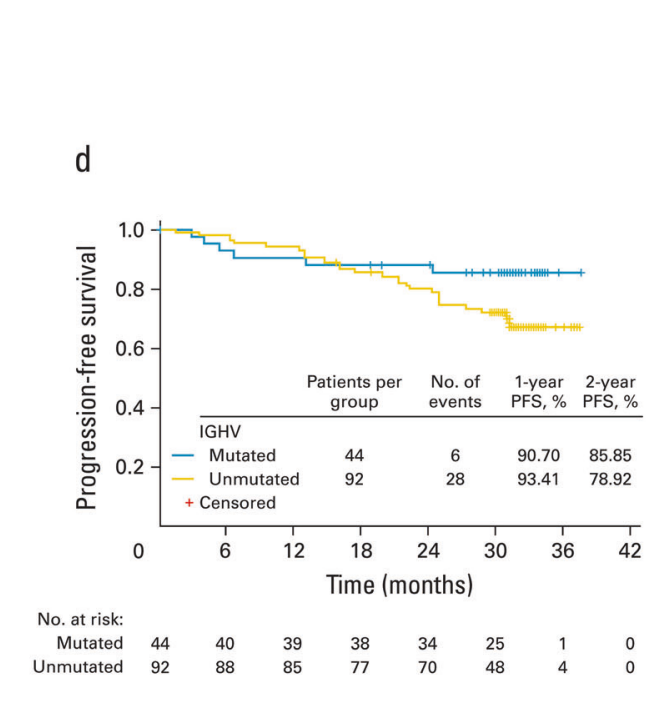

in CLL11 [11]. As with G-Clb in CLL11, no IRRs were fatal. Obinutuzumab administration in this subcohort of GREEN differed from that in CLL11 for the initial 1000
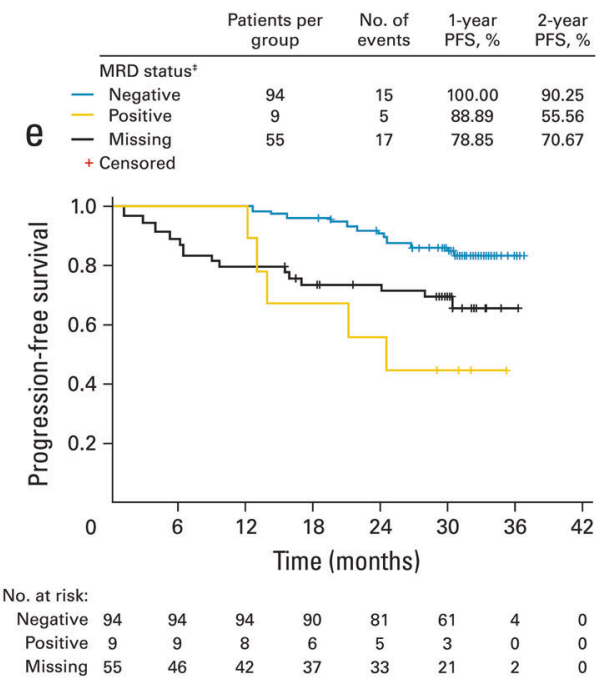

$\mathrm{mg}$, with an alternative split dose (25 mg on D1 at $12.5 \mathrm{mg} /$ $\mathrm{h}$, and $975 \mathrm{mg}$ on D2) [11]. Although split dosing did not appear to substantially reduce the incidence of IRRs vs 
CLL11, differences in study designs, treatment regimens and patient populations limit this comparison. Grade $\geq 3$ TLS and IRRs, and TLS as an SAE, were more frequently reported in unfit compared with fit patients, highlighting the need to monitor unfit patients and provide appropriate prophylaxis.

Fig. 1 Progression-free survival in patients receiving G-B in cohort 1 of GREEN: $\mathbf{a}$ in the overall study population; $\mathbf{b}$ in fit vs unfit patients; c by genomic aberrations, according to the hierarchical model [29]; d by IGHV mutation status; and e by MRD status at final response assessment in blood (intent-to-treat population). ${ }^{*} \mathrm{CIRS} \leq 6$ and $\mathrm{CrCl}$ $\geq 70 \mathrm{ml} / \mathrm{min} ;{ }^{\dagger} \mathrm{CIRS}>6$ and/or $\mathrm{CrCl}<70 \mathrm{ml} / \mathrm{min}$; ${ }^{\dagger}$ these data should be interpreted with caution as the subgroups are based on a study outcome, not baseline characteristics. There was also a low number of events $(n=20)$ and small number of MRD-positive patients $(n=9)$. The MRD-evaluable subgroup comprised patients who did not progress or die and had an MRD result available at the final response assessment in either blood or bone marrow $(n=105)$. The time window for MRD assessment was 77 to 168 days after last treatment. The MRD-'missing' subgroup included 54 patients. In addition, 1 out of 105 patients had a result available only in bone marrow and therefore came out as 'missing' in the blood population (therefore the 'missing' group in $\mathbf{e}$ is $n=55$ and not 54). Reasons for 'missing' included sample not taken $(n=20)$, shipment could not take place within $48 \mathrm{~h}$ $(n=18)$, measurement was outside the time window for the final response assessment $(n=9)$ or other reason $(n=7)$. Eleven patients with missing MRD status (in blood and bone marrow) progressed $(n=5)$ or died $(n=6)$ within the period since last treatment dose up to 168 days. CIRS Cumulative Illness Rating Scale, $\mathrm{CrCl}$ creatinine clearance, G-B obinutuzumab plus bendamustine, IGHV immunoglobulin heavy variable chain, MRD minimal residual disease, PFS progression-free survival
Grade $\geq 3$ neutropenia was reported in approximately half of patients, which was higher than that previously reported with G-Clb and R-B, but similar to that seen with G-B and G-FC [9, 11, 16]; importantly, grade $\geq 3$ neutropenia with $\mathrm{G}-\mathrm{B}$ in the present study was not associated with a marked increase in infections.

Seventeen deaths were reported in this cohort; 13 were due to AEs, of which 5 were considered related to G-B and 2 occurred post PD (Supplementary Table S3). No deaths were reported in the phase Ib GALTON study in fit patients with previously untreated CLL treated with G-B; however, this was a relatively small study (20 patients received G-B) undertaken at select sites [16]. Three deaths were reported among 102 patients in the phase II GIBB trial, but none were deemed related to treatment or CLL [15]. In CLL11, the percentage of patients with a fatal AE was lower with G-Clb (4\%) than with rituximabchlorambucil (R-Clb) or chlorambucil alone $(6 \%$ and $9 \%$, respectively) [11].

Patients receiving G-B achieved an ORR of $81.0 \%$, with $\mathrm{CR} / \mathrm{CRi}$ and $\mathrm{PR}$ in $34.8 \%$ and $46.2 \%$ of patients, respectively. Notably, response data were missing for $9.5 \%$ of patients (classed as non-responders). Despite some differences in response assessment (timing and criteria) between studies, response rates in all patients were broadly comparable with those reported for R-B in CLL10 (fit patients: ORR 96\%, CR 31\%) [5], G-Clb in CLL11 (unfit patients: ORR 78.4\%, CR 20.7\%) [11], and G-B in the GALTON (fit

Table 4 Disease characteristics and response (MRD or clinical) at the final response assessment in patients receiving G-B in cohort 1 of the GREEN study

\begin{tabular}{|c|c|c|c|}
\hline Factor, $n / N(\%)$ patients & MRD negative ${ }^{\mathrm{a}}$ (blood) & MRD negative ${ }^{\mathrm{a}}$ (bone marrow) & $\mathrm{CR} / \mathrm{Cri}^{\mathrm{b}}$ \\
\hline All patients & 94/104 (90.4) & $44 / 65(67.7)$ & $55 / 158(34.8)$ \\
\hline Binet stage A & $32 / 32(100)$ & $13 / 18(72.2)$ & 19/48 (39.6) \\
\hline Binet stage $B+C$ & $62 / 72(86.1)$ & $31 / 47(66.0)$ & $36 / 110(32.7)$ \\
\hline Disease bulk $\geq 5 \mathrm{~cm}$ & $57 / 64(89.1)$ & $27 / 39(69.2)$ & 29/95 (30.5) \\
\hline $\mathrm{ALC} \geq 50 \times 10^{9} / 1$ & $52 / 59(88.1)$ & $25 / 38(65.8)$ & 33/88 (37.5) \\
\hline $17 \mathrm{p}$ deletion & $3 / 6(50.0)$ & $2 / 3(66.7)$ & 2/11 (18.2) \\
\hline 11q deletion & $18 / 23(78.3)$ & $5 / 15(33.3)$ & $7 / 26(26.9)$ \\
\hline $12 q$ trisomy & $18 / 20(90.0)$ & $12 / 14(85.7)$ & $8 / 26(30.8)$ \\
\hline $13 q$ deletion & $32 / 32(100)$ & $13 / 19(68.4)$ & $22 / 52(42.3)$ \\
\hline CD38 positive & 49/55 (89.1) & $17 / 28(60.7)$ & $22 / 70(31.4)$ \\
\hline CD38 negative & $37 / 41(90.2)$ & $22 / 30(73.3)$ & $22 / 59(37.3)$ \\
\hline ZAP70 positive & $54 / 60(90.0)$ & $22 / 34(64.7)$ & $30 / 82(36.6)$ \\
\hline ZAP70 negative & 32/36 (88.9) & $17 / 24(70.8)$ & $14 / 47(29.8)$ \\
\hline$I G H V$ mutated & $31 / 31(100)$ & 12/17 (70.6) & $17 / 44(38.6)$ \\
\hline$I G H V$ unmutated & $58 / 68(85.3)$ & 28/44 (63.6) & 30/92 (32.6) \\
\hline
\end{tabular}

$A L C$ absolute lymphocyte count, $C R$ complete response, $C R i$ complete response with incomplete marrow recovery, $G-B$ obinutuzumab plus bendamustine, $I G H V$ immunoglobulin heavy variable chain, $M R D$ minimal residual disease

${ }^{a}$ Patients with evaluable (laboratory sample with a valid result at the final response assessment) MRD

${ }^{\mathrm{b}}$ Patients who achieved CR/CRi at the final response assessment in the intent-to-treat population 
patients: ORR 90\%, CR/CRi 45\%) [16] and GIBB (ORR 89.2\%; CR/CRi 49.0\%) [15] studies. Efficacy (response and PFS) was also similar to R-FC in CLL8 (fit patients) [3]. Importantly, response rates were similar between fit and unfit patients, and a high $C R$ rate was noted in patients with Binet stage $B+C$ CLL, showing that G-B is efficacious in this population. The high CR/CRi rate in patients with unmutated IGHV (34.8\%) was reassuring given the association between unmutated IGHV status and poor prognosis [20].

MRD status has been identified as a strong post-treatment prognostic factor after chemoimmunotherapy, with $\mathrm{MRD}^{-}$ independently associated with superior PFS and overall survival [21-24]. The sensitive quantification of MRD using flow cytometry in patients with CLL is well documented [2528]. $\mathrm{MRD}^{-}$in blood after G-B is expected to be associated with a favorable course during follow-up, as reported with GClb in CLL11 or R-FC in CLL8 [4, 11]. Despite the limited sample size and potential for bias, as patients were categorized based on outcome rather than baseline characteristics, the PFS data obtained so far in the present analysis are suggestive of a favorable prognosis in MRD-negative patients. The apparent discord between the high rate of $\mathrm{MRD}^{-}$but slightly lower than expected CR rates likely reflects the rigorous iwCLL assessment criteria [14] used for response classification and that patients with missing assessments within the required time window were down-classed.

Genomic factors, such as $11 \mathrm{q}$ and $17 \mathrm{p}$ chromosome deletions, mutated TP53, unmutated IGHV status, ZAP70 expression, and markers including increased serum $\beta_{2}$-microglobulin, are associated with poor prognosis [4, 29-34]. In GREEN, patients with an ALC $\geq 50 \times 10^{-9} / \mathrm{l}$, disease bulk $\geq 5 \mathrm{~cm}$, Binet stage $\mathrm{B}+\mathrm{C}$ or unmutated IGHV achieved high $\mathrm{MRD}^{-}$remission levels, while lower levels were seen in patients harboring $17 \mathrm{p}$ or $11 \mathrm{q}$ deletions. The low $\mathrm{MRD}^{-}$and response rates, and shorter PFS in patients with a 17p deletion, reflects the known poor prognosis of this subgroup and emphasizes that these patients need alternative treatment. As numbers in the GREEN analysis were relatively small, the promising $\mathrm{MRD}^{-}$data warrant further investigation of the G-B regimen.

In summary, G-B may represent a new option for previously untreated CLL patients regardless of fitness. Although the non-comparative design of GREEN prevents formal statistical analysis, frontline G-B appeared to have manageable toxicity in fit or unfit patients with CLL. Nonetheless, the TLS rate and associated fatalities highlights the need for careful risk assessment, prophylaxis and monitoring. The low rate of progression and excellent rates of $\mathrm{MRD}^{-}$with $\mathrm{G}-\mathrm{B}$ indicate that this combination is clinically active. Further follow-up is required to confirm these observations.

Acknowledgements The authors would like to thank the patients and their families, and the GREEN study investigators, coordinators, and nurses. Additionally, the authors would like to acknowledge the scientists and technicians at the German CLL Study Group laboratories who undertook the molecular and laboratory analyses, and Kerstin Trunzer (Roche) for her contribution to the minimal residual disease analyses. We would also like to thank Nicola Tyson (F. Hoffmann-La Roche Ltd) for her support and guidance with these analyses, as well as Corinna Miede (Accovion $\mathrm{GmBH}$, Marburg, Germany) who also provided invaluable support regarding the data. Editorial support under the direction of the lead author was provided by Cheryl Wright and Scott Malkin of Gardiner-Caldwell Communications (Macclesfield, UK), funded by F. Hoffmann-La Roche Ltd. GREEN was sponsored by F. Hoffmann-La Roche Ltd.

Funding This study was sponsored by F. Hoffmann-La Roche Ltd.

\section{Compliance with ethical standards}

Conflict of interest SS reports consulting fees, research funding, honoraria and membership of advisory committees from Roche. VL reports speaker's bureau fees, consulting fees, research funding, honoraria and membership of advisory committees from Roche; speaker's bureau fees, consulting fees, honoraria and membership of advisory committees from Janssen; speaker's bureau fees, honoraria and membership of advisory committees from Gilead and GSK; membership of advisory committees for AbbVie; and speaker's bureau fees from Mundipharma. RF reports speaker's bureau fees, consulting fees, honoraria and membership of advisory committees from Roche, Janssen, Celgene, AbbVie, Amgen and Novartis. SB reports consulting fees from Roche and AbbVie; research funding from Roche, AbbVie and Celgene; and honoraria from Roche, AbbVie and Beckton Dickinson. WK reports honoraria and participation in advisory boards for Roche and Mundipharma. CR reports research funding and membership of advisory committees from Roche and Celgene. FB reports consulting fees and honoraria from Roche, Novartis, Janssen, AbbVie, Gilead and Mundipharma; and research funding from Roche and Janssen. OI, EM, ET and DW report research funding from Roche. EG, LL, T Moore, T Morris and SR report employment from Roche.

Open Access This article is licensed under a Creative Commons Attribution 4.0 International License, which permits use, sharing, adaptation, distribution and reproduction in any medium or format, as long as you give appropriate credit to the original author(s) and the source, provide a link to the Creative Commons license, and indicate if changes were made. The images or other third party material in this article are included in the article's Creative Commons license, unless indicated otherwise in a credit line to the material. If material is not included in the article's Creative Commons license and your intended use is not permitted by statutory regulation or exceeds the permitted use, you will need to obtain permission directly from the copyright holder. To view a copy of this license, visit http://creativecommons. org/licenses/by/4.0/.

\section{References}

1. Tadmor T, Polliack A. Optimal management of older patients with chronic lymphocytic leukemia: some facts and principles guiding therapeutic choices. Blood Rev. 2012;26:15-23.

2. Thompson PA, Tam CS, O'Brien SM, Wierda WG, Stingo F, Plunkett W, et al. Fludarabine, cyclophosphamide, and rituximab treatment achieves long-term disease-free survival in IGHVmutated chronic lymphocytic leukemia. Blood. 2016;127:303-9.

3. Fischer K, Bahlo J, Fink AM, Goede V, Herling CD, Cramer P, et al. Long-term remissions after FCR chemoimmunotherapy in previously untreated patients with CLL: updated results of the CLL8 trial. Blood. 2016;127:208-15. 
4. Hallek M, Fischer K, Fingerle-Rowson G, Fink AM, Busch R, Mayer J, et al. Addition of rituximab to fludarabine and cyclophosphamide in patients with chronic lymphocytic leukaemia: a randomised, open-label, phase 3 trial. Lancet. 2010;376:1164-74.

5. Eichhorst B, Fink AM, Bahlo J, Busch R, Kovacs G, Maurer C, et al. First-line chemoimmunotherapy with bendamustine and rituximab versus fludarabine, cyclophosphamide, and rituximab in patients with advanced chronic lymphocytic leukaemia (CLL10): an international, open-label, randomised, phase 3, non-inferiority trial. Lancet Oncol. 2016;17:928-42.

6. Eichhorst B, Robak T, Montserrat E, Ghia P, Hillmen P, Hallek M, Buske C, ESMO Guidelines Committee. Chronic lymphocytic leukaemia: ESMO Clinical Practice Guidelines for diagnosis, treatment and follow-up. Ann Oncol. 2015;26(Suppl 5):v78-v84.

7. Martell RE, Peterson BL, Cohen HJ, Petros WP, Rai KR, Morrison VA, et al. Analysis of age, estimated creatinine clearance and pretreatment hematologic parameters as predictors of fludarabine toxicity in patients treated for chronic lymphocytic leukemia: a CALGB (9011) coordinated intergroup study. Cancer Chemother Pharmacol. 2002;50:37-45.

8. Shah N, Tam C, Seymour JF, Rule S. How applicable is fludarabine, cyclophosphamide and rituximab to the elderly? Leuk Lymphoma. 2015;56:1599-610.

9. Fischer $\mathrm{K}$, Cramer $\mathrm{P}$, Busch $\mathrm{R}$, Stilgenbauer $\mathrm{S}$, Bahlo J, Schweighofer CD, et al. Bendamustine combined with rituximab in patients with relapsed and/or refractory chronic lymphocytic leukemia: a multicenter phase II trial of the German Chronic Lymphocytic Leukemia Study Group. J Clin Oncol. 2011;29:3559-66.

10. Fischer K, Cramer P, Busch R, Böttcher S, Bahlo J, Schubert J, et al. Bendamustine in combination with rituximab for previously untreated patients with chronic lymphocytic leukemia: a multicenter phase II trial of the German Chronic Lymphocytic Leukemia Study Group. J Clin Oncol. 2012;30:3209-16.

11. Goede V, Fischer K, Busch R, Engelke A, Eichhorst B, Wendtner $\mathrm{CM}$, et al. Obinutuzumab plus chlorambucil in patients with CLL and coexisting conditions. N Engl J Med. 2014;370:1101-10.

12. Bosch F, Illmer T, Turgut M, Cortelezzi A, Lasserre SF, TruppelHartmann A, et al. Preliminary safety results from the Phase IIIb GREEN study of obinutuzumab (GA101) alone or in combination with chemotherapy for previously untreated or relapsed/refractory chronic lymphocytic leukemia (CLL). Blood. 2014;124:3345.

13. Howard SC, Jones DP, Pui CH. The tumor lysis syndrome. N Engl J Med. 2011;364:1844-54.

14. Hallek M, Cheson BD, Catovsky D, Caligaris-Cappio F, Dighiero G, Döhner H, et al. Guidelines for the diagnosis and treatment of chronic lymphocytic leukemia: a report from the International Workshop on Chronic Lymphocytic Leukemia updating the National Cancer Institute-Working Group 1996 guidelines. Blood. 2008;111:5446-56.

15. Danilov A, Yimer H, Boxer M, Di Bella N, Babu S, Li J, et al. Results of a phase II multicenter study of obinutuzumab plus bendamustine in pts with previously untreated chronic lymphocytic leukemia. Haematologica. 2017;102:71. Abstract P249

16. Brown JR, O'Brien S, Kingsley CD, Eradat H, Pagel JM, Lymp J, et al. Obinutuzumab plus fludarabine/cyclophosphamide or bendamustine in the initial therapy of CLL patients: the phase $1 \mathrm{~b}$ GALTON trial. Blood. 2015;125:2779-85.

17. Gazyva ${ }^{\circledR}$ (obinutuzumab) injection, for intravenous infusion. Full prescribing Information. Revised 2/2016.

18. Gazyvaro ${ }^{\circledast}$ (obinutuzumab) $1,000 \mathrm{mg}$ concentrate for solution for infusion. Summary of product characteristics. Last updated, 28/07/ 2016.

19. Freeman CL, Morschhauser F, Sehn L, Dixon M, Houghton R, Lamy T, et al. Cytokine release in patients with CLL treated with obinutuzumab and possible relationship with infusion-related reactions. Blood. 2015;126:2646-9.
20. Nabhan C, Raca G, Wang YL. Predicting prognosis in chronic lymphocytic leukemia in the contemporary era. JAMA Oncol. 2015;1:965-74.

21. Moreton P, Kennedy B, Lucas G, Leach M, Rassam SM, Haynes A, et al. Eradication of minimal residual disease in B-cell chronic lymphocytic leukemia after alemtuzumab therapy is associated with prolonged survival. J Clin Oncol. 2005;23:2971-9.

22. Böttcher S, Ritgen M, Fischer K, Stilgenbauer S, Busch RM, Fingerle-Rowson G, et al. Minimal residual disease quantification is an independent predictor of progression-free and overall survival in chronic lymphocytic leukemia: a multivariate analysis from the randomized GCLLSG CLL8 trial. J Clin Oncol. 2012;30:980-8.

23. Thompson PA, Wierda WG. Eliminating minimal residual disease as a therapeutic end point: working toward cure for patients with CLL. Blood. 2016;127:279-86.

24. Kovacs G, Robrecht S, Fink AM, Bahlo J, Cramer P, von Tresckow J, et al. Minimal residual disease assessment improves prediction of outcome in patients with chronic lymphocytic leukemia (CLL) who achieve partial response: comprehensive analysis of two phase III studies of the German CLL study group. J Clin Oncol. 2016;34:3758-65.

25. Rawstron AC, Kennedy B, Evans PA, Davies FE, Richards SJ, Haynes AP, et al. Quantitation of minimal residual disease levels in chronic lymphocytic leukemia using a sensitive flow cytometric assay improves the prediction of outcome and can be used to optimize therapy. Blood. 2001;98:29-35.

26. Moreno C, Villamor N, Colomer D, Esteve J, Giné E, Muntañola A, et al. Clinical significance of minimal residual disease, as assessed by different techniques, after stem cell transplantation for chronic lymphocytic leukemia. Blood. 2006;107:4563-9.

27. Raponi S, Della Starza I, De Propris MS, Del Giudice I, Mauro FR, Marinelli M, et al. Minimal residual disease monitoring in chronic lymphocytic leukaemia patients. A comparative analysis of flow cytometry and ASO IgH RQ-PCR. Br J Haematol. 2014;166:360-8.

28. Böttcher S, Stilgenbauer S, Busch R, Brüggemann M, Raff T, Pott C, et al. Standardized MRD flow and ASO IGH RQ-PCR for MRD quantification in CLL patients after rituximab-containing immunochemotherapy: a comparative analysis. Leukemia. 2009;23:2007-17.

29. Döhner H, Stilgenbauer S, Benner A, Leupolt E, Kröber A, Bullinger L, et al. Genomic aberrations and survival in chronic lymphocytic leukemia. N Engl J Med. 2000;343:1910-6.

30. Kröber A, Seiler T, Benner A, Bullinger L, Brückle E, Lichter P, et al. $\mathrm{V}(\mathrm{H})$ mutation status, CD38 expression level, genomic aberrations, and survival in chronic lymphocytic leukemia. Blood. 2002;100:1410-6.

31. Oscier DG, Gardiner AC, Mould SJ, Glide S, Davis ZA, Ibbotson $\mathrm{RE}$, et al. Multivariate analysis of prognostic factors in CLL: clinical stage, $\mathrm{IgVH}$ gene mutational status, and loss or mutation of the p53 gene are independent prognostic factors. Blood. 2002;100:1177-84.

32. Stilgenbauer S, Schnaiter A, Paschka P, Zenz T, Rossi M, Döhner $\mathrm{K}$, et al. Gene mutations and treatment outcome in chronic lymphocytic leukemia: results from the CLL8 trial. Blood. 2014;123:3247-54.

33. Rassenti LZ, Jain S, Keating MJ, Wierda WG, Grever MR, Byrd JC, et al. Relative value of ZAP-70, CD38, and immunoglobulin mutation status in predicting aggressive disease in chronic lymphocytic leukemia. Blood. 2008;112:1923-30.

34. Oscier D, Wade R, Davis Z, Morilla A, Best G, Richards S, et al. Prognostic factors identified three risk groups in the LRF CLL4 trial, independent of treatment allocation. Haematologica. 2010;95:1705-12. 